\title{
Molecular characterization of Nipah virus from Pteropus hypomelanus in Southern Thailand
}

\author{
Supaporn Wacharapluesadee ${ }^{*}$, Panumas Samseeneam², Mana Phermpool ${ }^{2}$, Thongchai Kaewpom', \\ Apaporn Rodpan', Pattarapol Maneeorn², Phimchanok Srongmongkol ${ }^{2}$, Budsabong Kanchanasaka² \\ and Thiravat Hemachudha ${ }^{1}$
}

\begin{abstract}
Background: Nipah virus (NiV) first emerged in Malaysia in 1998, with two bat species (Pteropus hypomelanus and P. vampyrus) as the putative natural reservoirs. In 2002, NiV lgG antibodies were detected in these species from Thailand, but viral RNA could not be detected for strain characterization. Two strains of NiV (Malaysia and Bangladesh) have been found in P. Iylei in central Thailand, although Bangladesh strain, the causative strain for the outbreak in Bangladesh since 2001, was dominant. To understand the diversity of NiV in Thailand, this study identified NiV strain, using molecular characterizations, from P. hypomelanus in southern Thailand.

Findings: Pooled bat urine specimens were collected from plastic sheet underneath bat roosts in April 2010, and then monthly from December 2010 to May 2011 at an island in southern Thailand. Five in 184 specimens were positive for NiV, using duplex nested RT-PCR assay on partial nucleocapsid fragment (357 bp). Whole sequences of nucleocapsid gene from four bats were characterized. All 5 partial fragments and 4 whole nucleocapsid genes formed a monophyletic with NiV-MY.

Conclusions: Our study showed that P. hypomelanus in southern Thailand and from Malaysia, a bordering country, harbored similar NiV. This finding indicates that NiV is not limited to central Thailand or P. lylei species, and it may be a source of inter-species transmission. This indicates a higher potential for a widespread NiV outbreak in Thailand. NiV surveillance in Pteropus bats, the major natural reservoirs, should be conducted continuously in countries or regions with high susceptibility to outbreaks.
\end{abstract}

Keywords: Nipah virus, Bats, Strain, Transmission, Southern, Thailand

\section{Background}

Nipah virus $(\mathrm{NiV})$ has been genetically characterized as Malaysia (NiV-MY) or Bangladesh (NiV-BD) strains, termed based on the country of their first outbreak, with fruit bats as the main reservoir [1]. Based on currently available complete $\mathrm{N}$ gene sequences, NiV-MY was obtained from Malaysia (Pteropus hypomelanus, P. vampyrus, sick pigs and patients) and Cambodia (P. lylei), while NiV-BD was obtained from patients from Bangladesh and India. Outbreak in Malaysia of NiV-MY in 1998

\footnotetext{
* Correspondence: spwa@hotmail.com

${ }^{1}$ World Health Organization Collaborating Centre for Research and Training on Viral Zoonoses, King Chulalongkorn Memorial Hospital, Faculty of Medicine, Chulalongkorn University, Bangkok, Thailand

Full list of author information is available at the end of the article
}

contributed to encephalitis with $39 \%$ fatality rate [2]. Recent encephalitis outbreak (most likely caused by NiV) in Southern Philippines (2014) originated from fruit bats, transmitted to horses and then to humans, which was attributable to either horse slaughter or horse meat consumption [3]. Secondary man-to-man spread was evident, with acute encephalitis, severe influenza-like illness, or meningitis ( $82 \%$ fatality). NiV-BD has been associated with the Indian (2001) and Bangladeshi (since 2001) outbreaks of encephalitis and respiratory distress syndrome, with a fatality rate of over $70 \%$ [4]. Infectivity and pathogenicity of the two strains may be different, as NiV-BD seems to be associated with higher incidence of respiratory disease. A study found higher level of oral shedding in ferrets 
infected with NiV-BD than with NiV-MY [5]. However, both strains caused similar respiratory tract lesions in Syrian hamsters [6]. On the other hand, disease does not develop in Pteropid bats, whether infected naturally or experimentally [7-9].

Serological studies have demonstrated evidences of $\mathrm{NiV}$ infection in multiple bat species, including frugivorous and insectivorous bats. However, viral isolation and molecular characterization was mostly only successful in Pteropus species [10]. NiV-MY was isolated from urine samples of $P$. hypomelanus [7] and $P$. vampyrus [11] from Malaysia. NiV-MY has also been found in other Pteropus bats from various countries; $P$. lylei in Cambodia, nucleocapsid (N) sequence of $1599 \mathrm{bp}$, shared $98 \%$ identity with the Malaysian patient (AF212302) [12] and P. vampyrus in Indonesia, matrix gene of 251 bp had $100 \%$ identity to NiV-MY in the Malaysian patient (AF212302) and 99.6\% homology with the Malaysian P. vampyrus bat (FN869553) [13]. P. giganteus is believed to be the source of NiV infection in India and Bangladesh via contaminated date palm

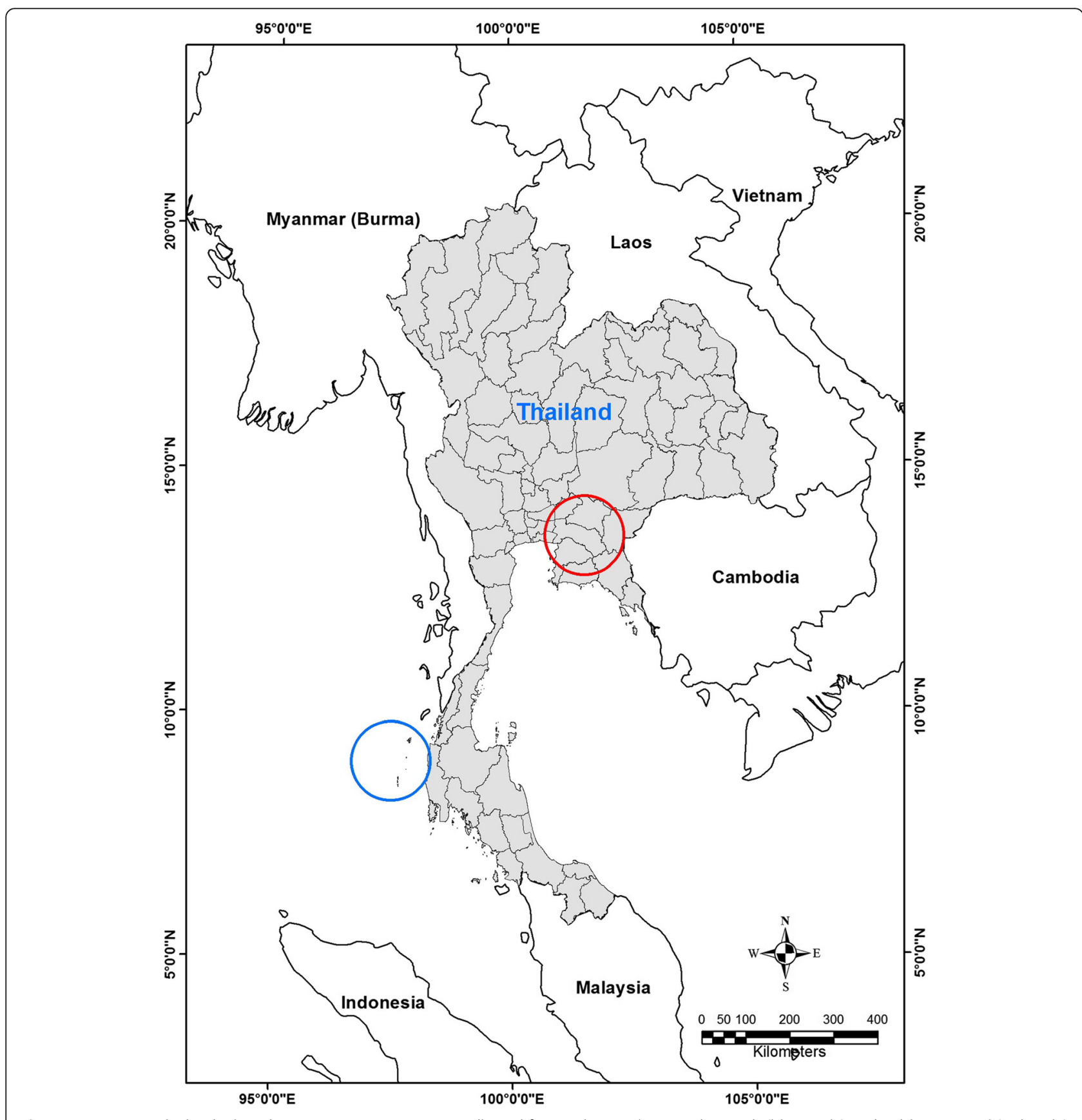

Fig. 1 Locations in Thailand where bat urines specimens were collected from P. hypomelanus at the south (blue circle) and P. Iylei at central (red circle) 
sap. NiV-BD has been detected from a liver homogenate of $P$. giganteus captured in India with its partial $\mathrm{N}$ gene (205 bp), showing $100 \%$ homology with NiV infected patients in India (FJ513078.1) and Bangladesh (AY988601) [14]. On the other hand $\mathrm{NiV}$ was detected in P. lylei from Thailand, with sequences (357 bp) sharing 98-99\% nucleotide homology with Bangladeshi patients (AY988601) [15], but there has been no report of an outbreak in humans or other animals.

The intensive surveillance of $\mathrm{NiV}$ in P. lylei in central Thailand has been conducted yearly since 2002. To date, NiV RNA was only detected in P. lylei among two other Pteropus species in Thailand. Fifty two (88.1\%) NiV-BD and 7 (11.9\%) NiV-MY were reported between 2002 and 2008 [15-17]. NiV IgG antibody has been found in P. hypomelanus and $P$. vampyrus from southern Thailand, but NiV RNA could not be successfully detected [16]. The objective of this study was to identify and characterize the strains of NiV found in P. hypomelanus, in southern Thailand.

\section{Methods}

Survey location was an island in Southern Thailand, a National Park, where only P. hypomelanus bats roosted in trees along the beach (Fig. 1). Numbers of bat population varied each month due to rain storms (Table 1). Bat urine samples were collected under roost trees with permission from the Department of National Parks, Wildlife and Plant Conservation (No. 0907.1/20713). The samples were collected once in April 2010, and then monthly from December 2010 to May 2011, using a plastic sheet protocol as previously described [18]. Two swabs of pooled bat urine collected from plastic sheet were stored in Lysis buffer (bioMérieux), transported on ice to the laboratory within $48 \mathrm{~h}$ and stored at $-80{ }^{\circ} \mathrm{C}$ until analysis. The nucleic acid was extracted from bat urine using the NucliSENS easyMAG ${ }^{\circ}$ extraction kit (bioMérieux). NiV RNA was first screened by duplex nested RT-PCR as previously described [17]. Only PCR positive specimens were further characterized for whole $\mathrm{N}$ gene by 3 additional PCR assays. All PCR positive specimens were directly sequenced on hemi-nested PCR products using an automated ABI PRISM 377 model sequencer. The sequence segments were assembled by BioEdit program [19]. Phylogenetic trees were generated by using maximum-likelihood method based on 357 bp (Fig. 2a) and 1599 bp (Fig. 2b) of $\mathrm{N}$ gene.

In addition, whole $\mathrm{N}$ gene sequences from six urine specimens of P. lylei, collected during 2010-2011 from central Thailand (see the map in Fig. 1), were included in the study. The sample collection and amplification methods were the same as for P. hypomelanus."

\section{Results}

Between 24 and 40 pooled bat urine samples were collected each trip (Table 1). NiV RNA was found in 5/184 (2.7 \%) samples; in April 2010 (2 of 28, $7.1 \%$ ), February 2011 ( 1 of 24, $4.1 \%$ ) and May 2011 (2 of 29, $6.9 \%$ ) (Table 1). All NiV sequences (357 bp) shared 98.6-100 \% identity with NiV-MY from the Malaysian P. hypomelanus (AF376747) [7]. Four of 5 PCR positive specimens were successfully sequenced further for the complete coding domains of the $\mathrm{N}$ gene (1599 bp). They showed 99.1-99.4 \% identity to NiV-MY from $P$. hypomelanus (Table 1). Whole $\mathrm{N}$ gene sequencing of sample no. 18254 (KT163257) with $100 \%$ identity to Malaysian $P$. hypomelanus $\mathrm{NiV}$ ( $357 \mathrm{bp}$ ) was not achieved due to low amount of viral RNA.

Phylogenetic analyses using maximum-likelihood methods from 357 and 1599 bp of $\mathrm{N}$ gene are shown in Fig. 1a and b respectively. Sequences were analyzed with available NiV sequences from bats, humans and pigs

Table 1 PCR testing of urine specimens for Nipah virus (partial fragment, 357 bp), categorized by month and year of collection, from a single colony of Pteropus hypomelanus from an island in southern Thailand

\begin{tabular}{|c|c|c|c|}
\hline Collected Dates & Population numbers ${ }^{a}$ & No. of positive/total ${ }^{\mathrm{b}}(\%)$ & Identity similarity to AF376747 (\%) \\
\hline & & & (GenBank number: identity/total nucleotides) \\
\hline \multirow[t]{2}{*}{ April 2010} & 800 & $2 / 28(7.1)$ & KT163247: 1584/1599 (99.1%) \\
\hline & & & KT163249: 1589/1599 (99.4 \%) \\
\hline December 2010 & 228 & $0 / 7(0)$ & - \\
\hline January 2011 & 218 & $0 / 30(0)$ & - \\
\hline February 2011 & 675 & $1 / 24(4.1)$ & KT163250: 1589/1599 (99.4 \%) \\
\hline March 2011 & 703 & 0/40 (0) & - \\
\hline April 2011 & 606 & 0/26 (0) & - \\
\hline \multirow[t]{2}{*}{ May 2011} & 555 & $2 / 29(6.9)$ & KT163248: 1588/1599 (99.3 \%) \\
\hline & & & KT163257: 357/357 (100 \%) \\
\hline Total & & $5 / 184(2.7)$ & \\
\hline
\end{tabular}

${ }^{\mathrm{a}}$ Bat population numbers were counted, using the bounded count method [22], by 10 skilled forest staff

${ }^{b}$ Pooled bat urine sample collected under the trees

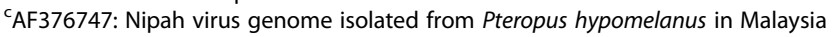


a

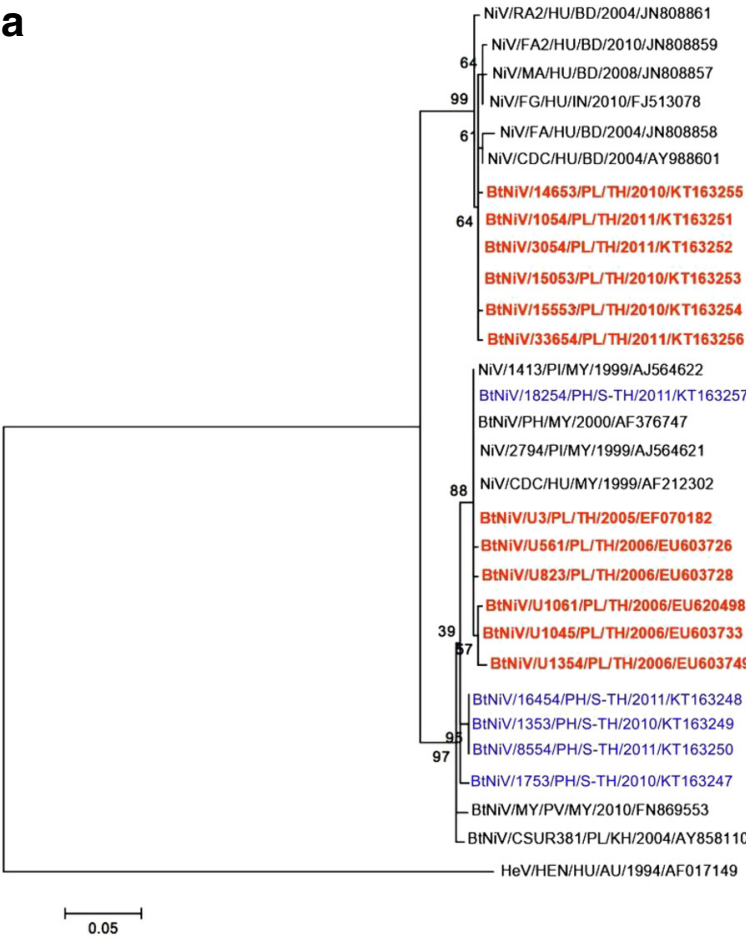

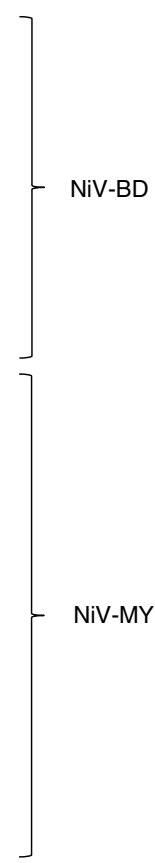

b

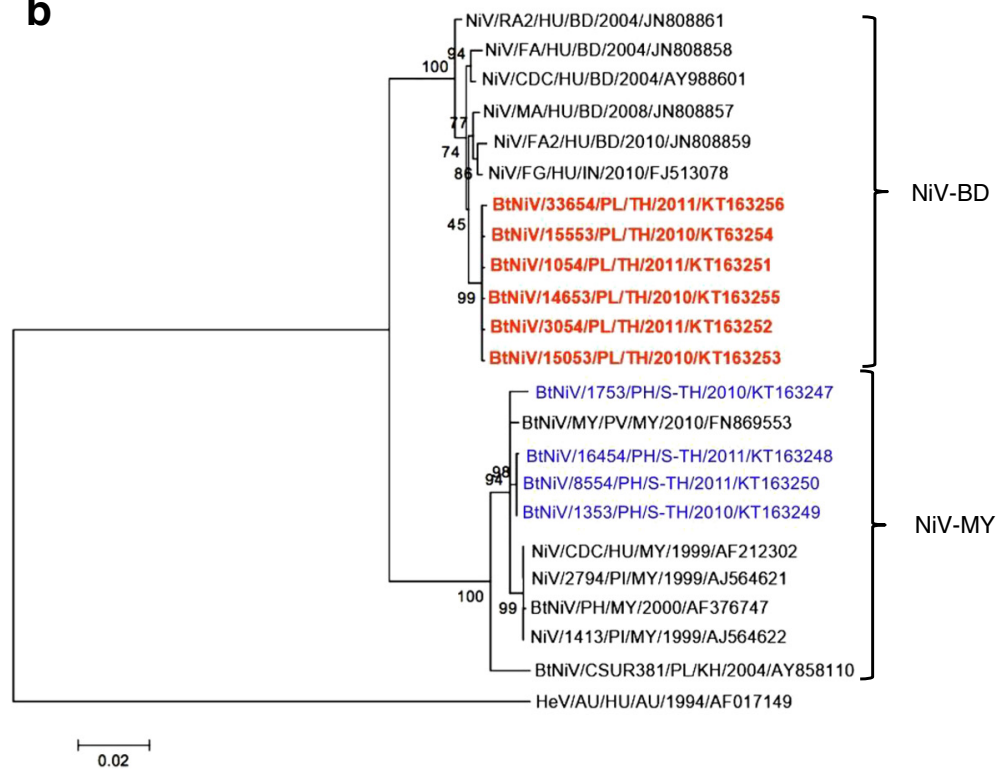

Fig. 2 Phylogenetic trees of Nipah virus (NiV) gene; a partial coding sequence (cds) of nucleocapsid ( $\mathrm{N}$ ) gene (357 bp), and $\mathbf{b}$ complete cds of N gene (1599 bp). Maximum-likelihood method was used to analyze phylogenetics, using sequences available in GenBank. NiV-MY partial N gene from P. lylei [15] and additional whole $\mathrm{N}$ gene sequences of NiV-BD obtained in 2010-2011 from urine of $P$. Iylei bats in Thailand (GenBank accession numbers KT163251KT163256) were included. Alignments were constructed by Multiple Alignment Fast Fourier Transform (MAFFT). Bootstrap values were determined using 1000 replicates via MEGA 6.06. The tree was then visualized using FigTree program, version 1.4.2. GenBank accession numbers are shown for corresponding sequences. Nivs found in P. hypomelanus and P. Iylei from Thailand are colored in blue and red respectively. Hendra virus (HeV) was used as the outgroup. Bt, Bat; HU, Human; PI, Pig; PV, Pteropus vampyrus; PH, P. hypomelanus; PL, P. Iylei; MY, Malaysia, BD, Bangladesh; IN, India; KH, Cambodia; TH, Thailand; S-TH, Southern Thailand 
in GenBank, and six additional whole $\mathrm{N}$ gene sequences of NiV-BD obtained in 2010-2011 from urine of P. lylei bats in central Thailand. The NiVs from P. hypomelanus in this study form a monophyletic clade with other NiVMY (Fig. 1a and b). All of NiV-MY (partial N gene, Fig. 2a) from $P$. lylei clustered with $P$. hypomelanus NiVs from southern Thailand (this study) and Malaysia.

Further, the similarity between the whole NiV-BD N gene from Thai $P$. lylei (KT163251- KT163256) and Bangladeshi patient (AY988601) was 99.1-99.2 \% (Fig. 2b). The nucleotide identity between NiV-N gene (205 bp) from $P$. giganteus in India [14] (JF899339, not included in the phylogenetic tree) and NiVs found in Thailand from $P$. hypomelanus (no.s 1353, 1753, 8554, 16454) and P. lylei (no.s 1054, 3054, 15053, 15553, 14653, 33654) were 97.1 and $99.5 \%$ respectively.

The visual alignment of the amino acid (aa) sequences was constructed from 533 aa of 1599 nucleotides of $\mathrm{N}$ gene open reading frame sequences (Fig. 3). The aa difference of $\mathrm{N}$ gene between strains NiV-MY and -BD sequences were found at 5 domains (positions 387, 505, 506, 508 and 521). The $\mathrm{N}$-aa sequences between sick pigs and patients from Malaysia were identical, while one aa sequence difference was observed in $\mathrm{NiV}$ isolate from P. hypomelanus in Malaysia (Fig. 3). There were 5-6 aa sequence differences between full-length $\mathrm{N}$ in $\mathrm{NiV}$ isolates from $P$. hypomelanus from southern Thailand and $P$. hypomelanus in Malaysia. However, they had only 1-2 aa sequence differences when compared with $\mathrm{NiV}$ isolate from $P$. vampyrus in Malaysia, and 4-5 aa sequence differences with $\mathrm{NiV}$ from $P$. lylei in Cambodia. Interestingly, $\mathrm{N}$-aa sequences among four NiV-BD isolates from P. lylei in central Thailand (GenBank accession nos. KT163251KT163254) and from Bangladeshi patient (GenBank accession no. JN808861) were identical (Fig. 3).

\section{Discussion and conclusion}

Whether $P$. hypomelanus or $P$. vampyrus bats were the spill-over hosts in the Malaysian outbreak has been unclear. Evidence in support of $P$. hypomelanus as the spill-over host is based on the near identical NiV sequence from this species to the other three NiVs isolated from human patients, and it was isolated shortly after the Malaysian outbreak [7]. In addition, the amino acid sequences obtained from NiV's whole genome differed from $\mathrm{NiV}$ from two infected pigs at merely 3 positions [20]. Nonetheless, NiV sequences from P. vampyrus bats have more differing nucleotides to the isolates from the patients during the outbreak than $\mathrm{NiV}$ from $P$. hypomelanus, 98 and 6 nucleotides, respectively.

Our study at the southern island confirmed the similarity between NiV-MY from P. hypomelanus from Thailand and the Malaysian outbreak (in humans and pigs), and was in accord with the finding from one $P$. hypomelanus from Tioman island, Malaysia, about $960 \mathrm{~km}$ from our surveyed site [7]. From our finding, it remains possible that $P$. hypomelanus is restricted to NiV-MY, as only this strain was found in the 5 pooled urine specimens positive for NiV.

\begin{tabular}{|c|c|c|c|c|c|c|c|c|c|c|c|c|c|c|c|c|c|c|c|c|c|c|c|c|}
\hline \multirow[b]{2}{*}{ NAME AND ACCESSION NO. } & \multicolumn{24}{|c|}{ Amino acid position (changed nucleotide) } \\
\hline & s & 30 & 155 & 188 & 191 & 211 & 318 & 380 & 387 & 406 & 429 & 414 & 432 & 436 & 450 & 457 & 481 & 502 & 505 & 506 & 508 & 511 & 518 & 521 \\
\hline NiV/CDC/HU/MY/1999/AF212302 & MY & $T(C)$ & I(A) & $E(A)$ & $T(A)$ & $Q(A)$ & I(A) & $N(A)$ & $D(G)$ & $S(G)$ & I(A) & $K(A)$ & $G(G)$ & I(A) & $M(A)$ & $N(A)$ & $\mathrm{S}(\mathrm{C})$ & $\mathrm{I}(\mathrm{T})$ & $R(G)$ & $\mathrm{T}(\mathrm{C})$ & $\mathrm{G}(\mathrm{G})$ & $E(G)$ & $L(A)$ & $A(G)$ \\
\hline NiV/2794/PI/MY/1999/AJ564621 & MY & . & . & . & . & . & . & . & . & . & . & . & . & . & . & . & . & . & . & . & . & . & . & . \\
\hline NiV/1413/PI/MY/1999/AJ564622 & MY & . & . & . & . & . & . & . & . & . & . & . & . & . & . & . & . & . & . & . & . & . & . & . \\
\hline BtNiV/PH/MY/2000/AF376747 & MY & I(T) & . & . & . & . & . & . & . & . & . & . & . & . & . & . & . & . & . & . & . & . & . & . \\
\hline BtNiV/MY/PV/MY/2010/FN869553 & MY & . & . & . & . & . & . & . & . & . & $V(A)$ & . & $E(A)$ & . & . & $\mathrm{D}(\mathrm{G})$ & . & . & . & . & . & . & . & . \\
\hline BtNiV/CSUR381/PL/KH/2004/AY858110 & MY & . & . & . & . & . & . & . & . & . & $V(A)$ & . & $E(A)$ & . & . & $D(G)$ & . & $T(C)$ & . & . & . & $G(G)$ & $P(C)$ & $T(A)$ \\
\hline BtNiV/1753/PH/S-TH/2010/KT163247 & MY & . & $V(G)$ & . & . & . & . & . & . & . & $V(A)$ & . & $E(A)$ & . & . & $D(G)$ & . & . & . & . & . & . & . & . \\
\hline BtNiV/16454/PH/S-TH/2011/KT163248 & MY & . & . & . & $A(G)$ & . & . & $S(G)$ & . & . & $V(A)$ & . & $E(A)$ & . & . & $\mathrm{D}(\mathrm{G})$ & . & . & . & . & . & . & . & . \\
\hline BtNiV/1353/PH/S-TH/2010/KT163249 & MY & . & . & . & . & . & . & $S(G)$ & . & . & $V(A)$ & . & $E(A)$ & . & . & $D(G)$ & . & . & . & . & . & . & . & . \\
\hline BtNiV/8554/PH/S-TH/2011/KT163250 & MY & . & . & . & . & . & . & $S(G)$ & . & . & $V(A)$ & . & $E(A)$ & . & . & $D(G)$ & . & . & . & . & . & . & . & . \\
\hline NiV/RA2/HU/BD/2004/JN808861 & BD & . & . & . & . & . & . & . & $N(A)$ & . & $V(A)$ & . & $E(A)$ & . & . & $\mathrm{D}(\mathrm{G})$ & . & . & $\mathrm{K}(\mathrm{A})$ & $D(A)$ & $\mathrm{R}(\mathrm{G})$ & $E(A)$ & . & $T(A)$ \\
\hline NiV/MA/HU/BD/2008/JN808857 & $\mathrm{BD}$ & . & . & . & . & . & . & . & $N(A)$ & . & $V(A)$ & . & $E(A)$ & . & . & $D(G)$ & . & . & $K(A)$ & $D(A)$ & $R(G)$ & . & . & $T(A)$ \\
\hline NiV/CDC/HU/BD/2004/AY988601.1 & BD & . & . & $\mathrm{D}(\mathrm{T})$ & . & . & . & . & $N(A)$ & . & $V(A)$ & . & $E(A)$ & . & . & $D(G)$ & . & . & $K(A)$ & $D(A)$ & $\mathrm{R}(\mathrm{G})$ & $E(A)$ & . & $T(A)$ \\
\hline NiV/FA2/HU/BD/2010/JN808859 & BD & . & . & . & . & . & $\mathrm{V}(\mathrm{G})$ & . & $N(A)$ & . & $V(A)$ & . & $E(A)$ & . & . & $D(G)$ & . & . & $\mathrm{K}(\mathrm{A})$ & $D(A)$ & $\mathrm{R}(\mathrm{G})$ & $E(A)$ & . & $T(A)$ \\
\hline NiV/FA/HU/BD/2004/JN808858 & $\mathrm{BD}$ & . & . & . & . & . & . & . & $N(A)$ & . & $V(A)$ & $N(T)$ & $E(A)$ & $M(G)$ & . & $D(G)$ & . & . & $\mathrm{K}(\mathrm{A})$ & $D(A)$ & $R(G)$ & $E(A)$ & . & $T(A)$ \\
\hline $\mathrm{NiV} / \mathrm{FG} / \mathrm{HU} / \mathrm{N} / 2010 / \mathrm{FJ} 513078$ & BD & . & . & . & . & $R(G)$ & . & . & $N(A)$ & . & $V(A)$ & . & $E(A)$ & . & . & $D(G)$ & . & . & $K(A)$ & $D(A)$ & $\mathrm{R}(\mathrm{G})$ & $E(A)$ & . & $T(A)$ \\
\hline BtNiV/1054/PL/TH/2011/KT163251 & BD & . & . & . & . & . & . & . & $N(A)$ & . & $V(A)$ & . & $E(A)$ & . & . & $D(G)$ & . & . & $K(A)$ & $D(A)$ & $\mathrm{R}(\mathrm{A})$ & $E(A)$ & . & $T(A)$ \\
\hline BtNiV/3054/PLTH/2011/KT163252 & BD & . & . & . & . & . & . & . & $N(A)$ & . & $V(A)$ & . & $E(A)$ & . & . & $D(G)$ & . & . & $\mathrm{K}(\mathrm{A})$ & $D(A)$ & $\mathrm{R}(\mathrm{A})$ & $E(A)$ & . & $T(A)$ \\
\hline BtNiV/15053/PL/TH/2010/KT163253 & BD & . & . & . & . & . & . & . & $N(A)$ & . & $V(A)$ & . & $E(A)$ & . & . & $D(G)$ & . & . & $K(A)$ & $D(A)$ & $\mathrm{R}(\mathrm{A})$ & $E(A)$ & . & $T(A)$ \\
\hline BtNiV/15553/PL/TH/2010/KT63254 & BD & . & . & . & . & . & . & . & $N(A)$ & . & $V(A)$ & . & $E(A)$ & . & . & $D(G)$ & . & . & $K(A)$ & $D(A)$ & $\mathrm{R}(\mathrm{A})$ & $E(A)$ & . & $T(A)$ \\
\hline BtNiV/14653/PL/TH/2010/KT163255 & BD & . & . & . & . & . & . & . & $N(A)$ & $N(A)$ & $V(A)$ & . & $E(A)$ & . & . & $D(G)$ & . & . & $K(A)$ & $D(A)$ & $\mathrm{R}(\mathrm{A})$ & $E(A)$ & . & $T(A)$ \\
\hline BtNiV/33654/PL/TH/2011/KT163256 & $\mathrm{BD}$ & & & & & & & & $N(A)$ & & $V(A)$ & & $E(A)$ & & $\mathrm{V}(\mathrm{C})$ & $D(G)$ & $L(T)$ & & $K(A)$ & $D(A)$ & $R(A)$ & $E(A)$ & & $T(A)$ \\
\hline
\end{tabular}

Fig. 3 Amino acid (aa) differences among available complete NiV N gene open reading frame sequences (ORF). A total of 533 aa sequences were compared among NiV isolates from P. hypomelanus (blue) and P. lylei (red) from Thailand and NiV aa sequences available in GenBank. "." Dots indicate sequences identical to AF212302; S, strain classification; MY, sequences from NiV-MY (Malaysia); BD, sequences from NiV-BD (Bangladesh); Bt, Bat; HU, Human; PI, Pig; PV, Pteropus vampyrus; PH, P. hypomelanus; PL, P. Iylei; MY, Malaysia, BD, Bangladesh; IN, India; KH, Cambodia; TH, Thailand; S-TH, Southern Thailand 
Unfortunately, there is limited data to support host specificity as only one NiV-MY from $P$. hypomelanus has been reported from Malaysia [7]. A comprehensive analysis of bats across their migratory routes in Indo-Australian region should be conducted to clearly understand the ecology and transmission of NiV in P. hypomelanus.

The co-circulation of NiV-BD and NiV-MY strains within population of $P$. lylei in central Thailand [15] remains a mystery. High percent similarity of $\mathrm{N}$ nucleotide sequences from $P$. lylei and $P$. giganteus, and $\mathrm{NiV}$ patients in Bangladesh and the identity of whole $\mathrm{N}$-aa among NiV isolates from $P$. lylei in Thailand and $\mathrm{NiV}$ patients in Bangladesh (Fig. 3), extrapolates the possibility of P. lylei bat in Thailand as a source of NiV infection to humans or other animals. The phylogenetic analysis on $357 \mathrm{bp}$ (Fig. 2a) revealed that NiV-MY in $P$. lylei from Thailand, and P. hypomelanus from Malaysia and Thailand clustered together. This finding introduces the possibility of NiV transmission between P. lylei and $P$. hypomelanus, in addition to the previously suggested transmission between $P$. vampyrus and $P$. lylei [11]. Furthermore, the higher percent similarity of NiV N-aa between $P$. hypomelanus in Thailand and $P$. vampyrus in Malaysia than P. hypomelanus in Malaysia, raise the possibility of transmission between $P$. hypomelanus and P. vampyrus. Molecular characterization of $\mathrm{NiV} \mathrm{N}$ and other genes, particularly those encoding the envelop glycoprotein from $P$. vampyrus in Thailand is required to investigate this possibility.

The alarming issue is that many bat species can harbor NiV. NiV-BD has been found in P. giganteus (India) [14], and P. lylei (Thailand) [16]. NiV-MY has been found in several bats species in many countries; $P$. hypomelanus (Malaysia [7] and Thailand [this study]), P. vampyrus (Malaysia [11] and Indonesia [13]), P. lylei (Cambodia [12] and Thailand [15]), Hipposideros larvatus (Thailand) [16], Taphozous species (Thailand) [15] and Rousettus amplexicaudatus (East Timor) [21]. The wide susceptibility of $\mathrm{NiV}$ to multiple host species allows for expedition of a larger-scale outbreak.

In conclusion, detecting NiV-MY strain in P. hypomelanus in southern Thailand, whose sequences share similarities to that identified in the Malaysian outbreak, and to $P$. lylei bats in central Thailand, suggests a potential role of $P$. hypomelanus as a possible source for inter-species transmission. This may result in the emergence of NiV infection to other animals and humans in both southern and central Thailand. Continuing surveillance of $\mathrm{NiV}$ infection in bats, humans, and pigs is vital for early detection and can potentially reduce the scale of an outbreak.

\section{Ethics approval and consent to participate}

Bat urine samples were collected with permission from the Department of National Parks, Wildlife and Plant
Conservation (No. 0907.1/20713). The urine specimens were collected under roost trees, no bat was caught for sample collection.

\section{Consent for publication \\ Not applicable.}

\section{Availability of data and materials}

The GenBank accession numbers for Nipah virus sequences reported in this paper are: KT163247- KT163250 for whole N gene and KT163257 for 357 bp partial N gene from Pteropus hypomelanus and KT163251- KT163256 for whole $\mathrm{N}$ gene from $P$. lylei.

\section{Abbreviations}

BD: Bangladesh; bp: base pairs; lgG: immunoglobulin G; MY: Malaysia; N fragment/ gene: nucleocapsid fragment/gene; NiV: Nipah virus; NiV-BD: NiV Bangladesh strain [NiV strain from the Bangladeshi and Indian outbreaks]; NiV-MY: NiV Malaysia strain [NiV strain from the Malaysian outbreak]; nt: nucleotides; PCR: Polymerase Chain Reaction; RNA: ribonucleic acid; RT-PCR: Reverse Transcription - Polymerase Chain Reaction.

\section{Competing interests}

The authors declare that they have no competing interests.

\section{Authors' contributions}

SW and TH participated in the design of the study and drafted the manuscript. SW, PS, MP, PM, and BK conducted sampling of bat urine samples. TK carried out the molecular genetic studies. AR participated in the sequence alignment. All authors read and approved the final manuscript.

\section{Acknowledgements}

We gratefully acknowledge the local support from the Thai Red Cross Society, Chulalongkorn University, Kasetsart University and the Department of National Parks Wildlife and Plant Conservation. We would also like to thank Ms. Siriporn Ghai for her intensive editing of the manuscript.

\section{Funding}

This study was supported by research grants from the Higher Education Research Promotion and National Research University Project of Thailand, Office of the Higher Education Commission (HR1 160A), the Ratchadaphiseksomphot Endowment Fund of Chulalongkorn University (RES560530148-HR), Health and Biomedical Science Research Program by the National Research Council of Thailand (NRCT) and Health System Research Institute (HSRI), the Research Chair Grant and the Cluster and Program Management Office (CPMO), from the National Science and Technology Development Agency (NSTDA), Thailand.

\section{Author details}

${ }^{1}$ World Health Organization Collaborating Centre for Research and Training on Viral Zoonoses, King Chulalongkorn Memorial Hospital, Faculty of Medicine, Chulalongkorn University, Bangkok, Thailand. ${ }^{2}$ Department of National Parks, Wildlife and Plant Conservation, Bangkok, Thailand.

Received: 29 December 2015 Accepted: 21 March 2016

Published online: 25 March 2016

References

1. Rota PA, Lo MK. Molecular virology of the henipaviruses. Curr Top Microbiol Immunol. 2012;359:41-58.

2. Chua KB. Nipah virus outbreak in Malaysia. J Clin Virol. 2003;26:265-75.

3. Ching PKG, de los Reyes VC, Sucaldito MN, Tayag E, Columna-Vingno AB, Malbas Jr FF, et al. Outbreak of Henipavirus Infection, Philippines, 2014. Emerg Infect Dis. 2015;21:328-31.

4. Luby SP, Hossain MJ, Gurley ES, Ahmed BN, Banu S, Khan SU, et al. Recurrent zoonotic transmission of Nipah virus into humans, Bangladesh, 2001-2007. Emerg Infect Dis. 2009;15:1229-35. 
5. Clayton BA, Middleton D, Bergfeld J, Haining J, Arkinstall R, Wang L, et al. Transmission routes for Nipah virus from Malaysia and Bangladesh. Emerg Infect Dis. 2012;18:1983-93.

6. Baseler L, de Wit E, Scott DP, Munster VJ, Feldmann H. Syrian hamsters (Mesocricetus auratus) oronasally inoculated with a Nipah virus isolate from Bangladesh or Malaysia develop similar respiratory tract lesions. Vet Pathol. 2015;52:38-45.

7. Chua KB, Koh CL, Hooi PS, Wee KF, Khong JH, Chua BH, et al. Isolation of Nipah virus from Malaysian Island flying-foxes. Microb Infect. 2002;4:145-51.

8. Halpin $\mathrm{K}$, Hyatt AD, Fogarty R, Middleton D, Bingham J, Epstein JH, et al. Pteropid bats are confirmed as the reservoir hosts of henipaviruses: a comprehensive experimental study of virus transmission. Am J Trop Med Hyg. 2011;85:946-51.

9. Rahman SA, Hassan L, Epstein JH, Mamat ZC, Yatim AM, Hassan SS, et al. Risk factors for Nipah virus infection among pteropid bats, Peninsular Malaysia. Emerg Infect Dis. 2013;19:51-60.

10. Simons RR, Gale P, Horigan V, Snary EL, Breed AC. Potential for Introduction of Bat-Borne Zoonotic Viruses into the EU: A Review. Viruses. 2014;6:2084-121.

11. Rahman SA, Hassan SS, Olival KJ, Mohamed M, Chang LY, Hassan L, et al. Characterization of Nipah virus from naturally infected Pteropus vampyrus bats, Malaysia. Emerg Infect Dis. 2010;16:1990-3.

12. Reynes JM, Counor D, Ong S, Faure C, Seng V, Molia S, et al. Nipah virus in Lyle's flying foxes, Cambodia. Emerg Infect Dis. 2005;11:1042-7.

13. Sendow I, Ratnawati A, Taylor T, Adjid RM, Saepulloh M, Barr J, et al. Nipah virus in the fruit bat Pteropus vampyrus in Sumatera, Indonesia. PLoS One. 2013:8:e69544. doi:10.1371/journal.pone.0069544.

14. Yadav PD, Raut CG, Shete AM, Mishra AC, Towner JS, Nichol ST, et al. Detection of Nipah virus RNA in fruit bat (Pteropus giganteus) from India. Am J Trop Med Hyg. 2012;87:576-8.

15. Wacharapluesadee S, Ngamprasertwong T, Kaewpom T, Kattong P, Rodpan A, Wanghongsa S, et al. Genetic characterization of Nipah virus from Thai fruit bats (Pteropus lylei). Asian Biomedicine. 2013;7:813-9.

16. Wacharapluesadee S, Lumlertdacha B, Boongird K, Wanghongsa S, Chanhome L, Rollin P, et al. Bat Nipah virus, Thailand. Emerg Infect Dis. 2005:11:1949-51.

17. Wacharapluesadee $S$, Hemachudha T. Duplex nested RT-PCR for detection of Nipah virus RNA from urine specimens of bats. J Virol Methods. 2007;141:97-101.

18. Chua KB. A novel approach for collecting samples from fruit bats for isolation of infectious agents. Microb Infect. 2003:5:487-90.

19. Hall TA. BioEdit: a user-friendly biological sequence alignment editor and analysis program for Windows 95/98/NT. Nucleic Acids Symp Ser. 1999;41:95-8.

20. AbuBakar S, Chang LY, Ali AR, Sharifah SH, Yusoff K, Zamrod Z. Isolation and molecular identification of Nipah virus from pigs. Emerg Infect Dis. 2004:12:2228-30.

21. Breed AC, Meers J, Sendow I, Bossart KN, Barr JA, Smith I, et al. The distribution of henipaviruses in Southeast Asia and Australasia: Is Wallace's line a barrier to Nipah virus. PLoS One. 2013;8:e61316. doi:10.1371/journal. pone.0061316.

22. Wacharapluesadee S, Boongird K, Wanghongsa S, Ratanasetyuth N, Supavonwong P, Saengsen D, et al. A longitudinal study of the prevalence of Nipah virus in Pteropus lylei bats in Thailand: evidence for seasonal preference in disease transmission. Vector Borne Zoonotic Dis. 2010;10:183-90.

\section{Submit your next manuscript to BioMed Central and we will help you at every step:}

- We accept pre-submission inquiries

- Our selector tool helps you to find the most relevant journal

- We provide round the clock customer support

- Convenient online submission

- Thorough peer review

- Inclusion in PubMed and all major indexing services

- Maximum visibility for your research

Submit your manuscript at www.biomedcentral.com/submit

) Biomed Central 